\title{
Prevalencia de infección tuberculosa \\ en escolares de la ciudad de Tijuana, México
}

\author{
Rafael Laniado-Laborín, M.C., M.S.P.,(1) Noemí Cabrales-Vargas, M.C., (2) \\ Graciano López-Espinoza, M.C., ${ }^{(2)}$ José Luis Lepe-Zúñiga, M.C., (3) \\ Sergio Quiñonez-Moreno, M.C., ${ }^{(3)}$ Claudia E. Rico-Vargas, M.C. ${ }^{(3)}$
}

\section{Laniado-Laborín R, Cabrales-Vargas $\mathbf{N}$, López-Espinoza G, Lepe-Zúñiga JL, Quiñonez-Moreno S, Rico-Vargas CE. \\ Prevalencia de infección tuberculosa en escolares de la ciudad de Tijuana, México. Salud Publica Mex I998;40:47-52.}

\section{Resumen}

Objetivo. Determinar la prevalencia de infección tuberculosa en escolares de la ciudad de Tijuana, México. Material y métodos. Se seleccionó aleatoriamente una muestra a partir del registro escolar municipal y se incluyeron I | $3 \mid$ estudiantes de primaria y secundaria. A todos se les aplicó una dosis de tuberculina 5TU (Mantoux). Los sujetos con induración $\geq 10 \mathrm{~mm}$ se clasificaron como reactores positivos. Resultados. El estudio demostró que entre los sujetos participantes la prevalencia global de reactores positivos fue de $57 \%$. La proporción de reactores positivos fue significativamente mayor en el grupo de sujetos inmunizados con BCG que en el grupo de no inmunizados ( 59.7 vs $45.6 \% ; p<0.00 I)$. No se encontró una correlación significativa entre el diámetro de la induración y la edad de aplicación de la BCG. Conclusiones. Estos hallazgos demuestran que la prevalencia de infección tuberculosa es extremadamente alta en la ciudad de Tijuana y tiene importantes implicaciones si se considera como una evidencia básica en el proceso para el control de la tuberculosis en la región.

Palabras clave: micobacteriosis; prevalencia; niño; adolescencia; México

\author{
Laniado-Laborín R, Cabrales-Vargas $\mathbf{N}$, \\ López-Espinoza G, Lepe-Zúñiga JL, \\ Quiñonez-Moreno S, Rico-Vargas CE. \\ Prevalence of tuberculosis infection \\ in schoolchildren from Tijuana, Mexico. \\ Salud Publica Mex 1998;40:47-52.
}

\section{Abstract}

Objective. To determine the prevalence of tuberculosis infection in school children from Tijuana, Mexico. Materials and methods. A study sample was randomly chosen from the municipal school registry and I $|3|$ elementary and high school children were included. All received one dosis of PPD 5 TU (Mantoux). Subjects with induration $\geq 10 \mathrm{~mm}$ were considered positive reactors. Results. The overall prevalence of positive reactors was $57 \%$. The proportion of positive reactors was significantly higher among BCGimmunized subjects than in non-immunized individuals (59.7 vs $45.6 \% ; p<0.00$ I). Correlation was not significant between age of immunization with BCG and diameter of induration. Conclusions. The prevalence of tuberculosis infection in Tijuana is extremely high; this fact has important implications in the control of tuberculosis in this region.

Key words: mycobacterium infections; prevalence; child; adolescence; Mexico

(I) Hospital General de Tijuana, Secretaría de Salud (SSA); Facultad de Medicina-Tijuana, Universidad Autónoma de Baja California, México.

(2) Hospital General de Tijuana, SSA, México.

(3) Facultad de Medicina-Tijuana, Universidad Autónoma de Baja California, México. 
A pesar de los esfuerzos en el ámbito internacional A para lograr el control de la tuberculosis (TB), esta infección sigue siendo la de mayor prevalencia en el mundo; se estima que, aproximadamente, una tercera parte de la población mundial se encuentra infectada por Mycobacterium tuberculosis. ${ }^{1}$

No existen informes recientes en la literatura sobre la prevalencia global de infección tuberculosa en la República mexicana. Olvera y colaboradores, en una recopilación de cinco encuestas realizadas en el estado de Jalisco en sujetos de 5-9 años encontraron una prevalencia de $19.74 \%{ }^{2}$ Una encuesta tuberculínica más reciente, también del estado de Jalisco, en niños de educación primaria (mediana de edad 6.7 años), detectó una prevalencia de reactores positivos al PPD 2TU de $6.8 \%$ y un riesgo anual de infección de $0.82 \% .{ }^{3}$ La Organización Mundial de la Salud estima que el riesgo anual de infección para América Latina es de $0.5-1.5 \% .{ }^{4}$ Aun cuando se utilice el estimado más bajo de $0.5 \%$ de riesgo de infección anual, con una población de 88 millones de mexicanos, se considera que ocurrirán 440000 infecciones anualmente, y que deben existir de 18 a 26 millones de mexicanos infectados con M. tuberculosis. ${ }^{5}$

El objetivo de la investigación fue determinar la prevalencia de infección tuberculosa por medio de una encuesta cutánea con tuberculina en escolares de la ciudad de Tijuana.

\section{Material y métodos}

De acuerdo con las cifras del censo de población más reciente, la ciudad de Tijuana tiene una población de 1237000 habitantes, y se encuentra urbanizada en una zona central y 412 colonias. Se localiza en la región noroccidental de nuestro país en el estado de Baja California. Su puerto fronterizo con los Estados Unidos de América (EUA) registra el mayor número de cruces legales (60 millones en 1996) e ilegales de toda la frontera entre México y los EUA, representando un foco de atracción para inmigrantes (y emigrantes potenciales), al grado de que aquéllos han llegado a constituirse en el segmento de mayor número $(52.5 \%)$ y causa del crecimiento de la población de la ciudad ( $2.79 \%$ anual), contribuyendo a un crecimiento demográfico explosivo; ${ }^{6}$ esto ha favorecido el desarrollo de condiciones insalubres en algunas áreas de la ciudad debido principalmente al encarecimiento de los servicios urbanos en todos los niveles.

Después de obtener la autorización de la Secretaría de Salud y del Departamento de Servicios Educativos y Pedagógicos del estado de Baja California, se procedió al muestreo aleatorio y por conglomerados; por medio de una lista de números aleatorios, se seleccionaron once escuelas primarias y cuatro secundarias del registro escolar municipal de la ciudad de Tijuana. Las escuelas constituyeron las unidades de muestreo a partir de las cuales se incluyeron grupos de primero y sexto año de primaria, y de tercero de secundaria.

Durante un periodo de seis semanas (mayo-junio 1996), 1143 escolares fueron sometidos a una prueba tuberculínica; 12 de ellos (1.04\%) fueron excluidos del análisis por no poderles realizar la lectura, pues faltaron a clase por dos días consecutivos. Los restantes 1131 escolares constituyen así nuestra población de estudio.

Un requisito indispensable para la inclusión de los escolares en la investigación fue el consentimiento informado por escrito de los padres de los sujetos. En el formato de autorización se solicitó además información demográfica básica; a partir de la cartilla de inmunizaciones se obtuvo información sobre historia de BCG (edad en la que fue inmunizado y recibió refuerzo, en su caso). En todos los sujetos se determinó al momento de la aplicación de la prueba tuberculínica la presencia o ausencia de cicatriz por BCG.

El personal que participó en la aplicación y lectura de las pruebas fue adiestrado en dicha técnica durante un estudio piloto por uno de los autores con experiencia en encuestas cutáneas similares. ${ }^{7}$

Se aplicó $0.1 \mathrm{ml}$ de PPD-S 5TU (lote CT68; Tubersol, ${ }^{\oplus}$ Connaught, Ontario, Canadá), por medio de la técnica estándar de Mantoux ${ }^{8}$ en la cara anterior del antebrazo izquierdo. El diámetro máximo de induración se midió 48 horas después utilizando el método del bolígrafo para determinar los bordes de la induración. ${ }^{9}$ Se registró el diámetro en mm en todos los casos, y en aquellos en que no se palpó induración se consignó como de $0 \mathrm{~mm}$. En los casos en que el sujeto no asistió a la escuela el día de la lectura, se intentó realizarla al día siguiente.

Durante toda la intervención se mantuvo un estricto control de calidad tanto en la aplicación como en la lectura de la prueba, verificando que la aplicación fuera correcta, y efectuando la lectura por consenso en caso de duda.

En todos los sujetos se investigó la presencia de reacciones adversas locales o sistémicas a la tuberculina.

A todos los participantes se les entregó una notificación dirigida a sus padres con los resultados de la prueba cutánea, incluyendo una sencilla explicación del significado de una prueba tuberculínica positiva o negativa, según el caso. Los reactores positivos fueron referidos a alguna de las instituciones del Sector Salud (o con su médico particular si así lo preferían) para que se descartara enfermedad tuberculosa. 
Se clasificó al sujeto como reactor positivo cuando el diámetro de induración a la tuberculina era $>10 \mathrm{~mm}$; los sujetos con diámetro de induración $<10 \mathrm{~mm}$ se clasificaron como negativos.

Tomando como base un riesgo anual de infección de $1 \%$, una región crítica de 0.05 , y poder de 0.80 , el cálculo del tamaño muestral indicó que deberían incluirse un mínimo de 1056 sujetos.

Se utilizaron como pruebas de significancia estadística las pruebas de $t$ y de ANDEVA en la comparación de medias de variables continuas con distribución normal (p.e., la edad); como el diámetro de la reacción a la tuberculina no tiene una distribución normal, para el análisis de esta variable se utilizaron las prueba $U$ de Mann-Whitney y de Wilcoxon; las medias se presentan indicando además el error estándar de la misma. En la comparación de proporciones se empleó la prueba de $X^{2}$ con corrección de Yates y la de Mantel-Haenzel para resultados categóricos.

Se llevó a cabo un análisis de correlación y de regresión lineal múltiple con el fin de determinar si existía alguna relación entre el diámetro de la induración (variable dependiente) y el resto de las variables.

El valor de $p<0.05$ fue utilizado para indicar significancia estadística. La información fue analizada utilizando el paquete estadístico comercial SPSS/PC+ (Statistical Package for the Social Sciences, SPSS Inc., Chicago Ill).

\section{Resultados}

La media de edad fue de $11.43+0.10$ años (IC 95\% 11.23, 11.63; rango 5-17 años); la mediana fue de 12 años. Quinientos ochenta de los 1131 sujetos (51.3\%) eran varones.

Se notificaron 35 diferentes lugares de origen; $63.9 \%$ de los escolares nacieron y han vivido siempre en la ciudad de Tijuana; otros lugares de origen informados con frecuencia fueron el Distrito Federal $(6.8 \%)$, los EUA (5.9\%), y los estados de Jalisco (5.5\%) y Sinaloa (3.2\%).

Fue posible establecer el antecedente de inmunización con BCG en 1112 sujetos $(98.3 \%)$; de éstos, 932 (83.81\%) habían sido inmunizados, y 180 (16.18\%) nunca la habían recibido. Tan sólo en 67 escolares (5.9\%) se notificó revacunación con BCG. No se encontró una diferencia significativa en el grado de cobertura cuando los sujetos se clasificaron por sexo (82.5\% de los varones habían sido inmunizados vs $85.2 \%$ de las mujeres; $X^{2}=1.58 ; p=0.20 ;$ IC 95\% 0.89,1.69). La edad promedio de aplicación de la BCG fue a los $1.89+0.10$ años. El intervalo promedio entre la edad de inmunización con BCG, y la edad de aplicación de la prueba tuberculínica, fue de $9.31+0.17$ años (rango 0-17 años).

La prevalencia de reactores positivos para toda la muestra, incluyendo 19 sujetos en quienes se desconocía el antecedente de inmunización con BCG, fue de $57.02 \%$ (645 reactores positivos de un total de 1131 sujetos). La figura 1 muestra la distribución de los sujetos de acuerdo con el diámetro de induración a la tuberculina.

Los varones presentaron una proporción significativamente menor de reactores positivos que las mujeres ( 52.8 vs 61.5\%; $X^{2}=8.86 ; p=0.003$; IC 95\% 1.15, 1.69).

A pesar de que el tiempo promedio de residencia en la ciudad de los sujetos nacidos fuera de Tijuana $(6.68+0.19$ años) fue significativamente menor que el de los nativos $(11.20+0.13$ años; $\mathrm{t}=19.6 ; p<0.001$; IC $95 \%-4.963,-4.064)$, no se encontró una diferencia significativa en la proporción de reactores positivos al comparar estos dos subgrupos (57.8 vs $55.7 \%$, respectivamente; $X^{2}=0.48 ; p=0.49$ IC; $95 \% 1.11,1.81$ ).

No se encontraron diferencias significativas en la proporción de reactores positivos al agrupar a los sujetos por plantel escolar (comparación entre escuelas, por grado escolar), ni tampoco al clasificarlos por el tipo de escuela, pública $(57.6 \%)$ o privada $(56.6 \%$; $X^{2}=0.06 ; p=0.80$ IC; $\left.95 \% 0.68,1.21\right)$.

El cuadro I muestra el número y edad promedio de los sujetos, la proporción de varones, y la proporción de reactores positivos, por grado escolar.

El cuadro II muestra la proporción de reactores positivos y negativos de acuerdo con el antecedente de inmunización con BCG. La proporción de reactores

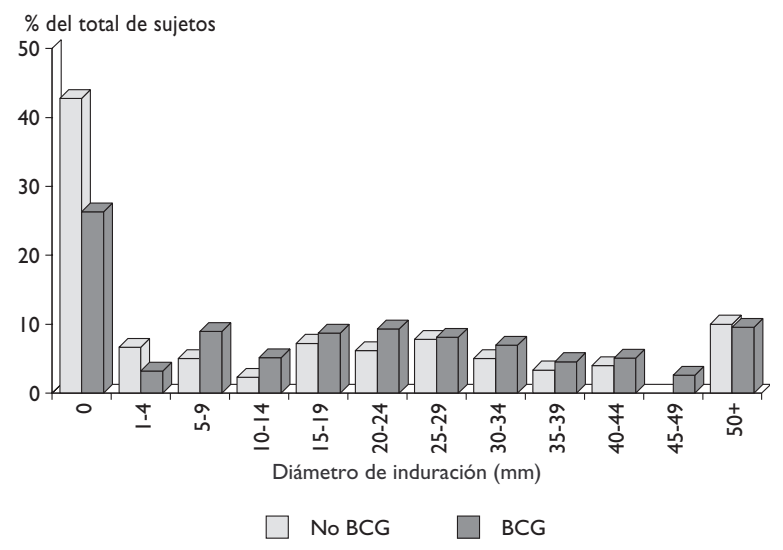

Figura I. Distribución de los reactores a la TUBERCULINA DE ACUERDO CON EL ANTECEDENTE DE INMUNIZACIÓN CON BCG 
positivos en el grupo de sujetos inmunizados es significativamente mayor que la del grupo de sujetos no inmunizados $\left(X^{2}=12.27\right.$; $p<0.001$; IC 95\% 1.28, 2.44).

$\mathrm{Al}$ analizar por grado escolar, la proporción de reactores positivos en el primer año de primaria es virtualmente idéntica en escolares inmunizados y no inmunizados con BCG (46.4 vs 47.7\%, respectivamente; $X^{2}=0.03 ; p=0.86$; IC 95\% 0.87, 1.06); para el tercer año de secundaria, $69.7 \%$ de los sujetos inmunizados son reactores positivos vs $49.9 \%$ de los no inmunizados $\left(X^{2}=12.2 ; p<0.001 ;\right.$ IC $\left.95 \% 1.27,2.28\right)$.

Entre los reactores positivos el diámetro de induración de los sujetos inmunizados fue en promedio de $32.1+0.74 \mathrm{~mm}$ vs $33.6+2.0 \mathrm{~mm}$ para los no inmunizados $(U=21$ 702.5; $p=0.48$; IC 95\% -5.606, 2.623$)$; entre los reactores negativos el diámetro promedio de induración fue de $1.86+0.14 \mathrm{~mm}$ en los escolares inmunizados vs $0.94+0.20 \mathrm{~mm}$ en los no inmunizados $(U=15$ $548.5 ; p=0.04$; IC 95\% 0.321,1.510).

$\mathrm{Al}$ analizar la relación entre el diámetro de induración (variable dependiente) y el resto de las variables, la edad del sujeto fue la única que mostró un coeficiente de correlación débil, pero significativo $(r=0.16 ; p<0.001)$. La edad de aplicación de la BCG no mostró una correlación significativa $(r=0.004 ; p=0.92)$. Esto se corroboró en el análisis de regresión múltiple, donde únicamente logran entrar en la ecuación ( $P I N=0.05)$ las variables antecedente de $B C G(B=0.08$; $p=0.003)$ y edad del sujeto $(\Omega=0.17 ; p<0.001$; cuadro III).

Entre los sujetos de estudio, 29 de ellos (2.6\%) presentaron pequeñas pápulas sobre la induración; ningún caso presentó vesículas o flictenas; en ocho casos $(0.7 \%)$ se informaron síntomas sistémicos (fiebre, mialgias). No se observaron reacciones anafilácticas.

\section{Discusión}

La prevalencia de infección tuberculosa se define como la proporción de individuos infectados en una población en un momento determinado. Se basa en la reactividad a la tuberculina, y usualmente se expresa como un porcentaje de los reactores a la aplicación de la misma. ${ }^{10}$

Mediante esta encuesta de tuberculina en una muestra representativa de escolares de la ciudad de Tijuana, la prevalencia de infección global fue de $57 \%$; ésta es considerablemente mayor a la informada en encuestas similares llevadas a cabo en otras regiones del país. ${ }^{2,3}$ Aun cuando se incluyeran en el análisis únicamente los sujetos no inmunizados con BCG para eliminar cualquier efecto atribuible a esta inmunización sobre la reactividad a la tuberculina, la prevalencia de

\section{Cuadro I}

EdAD, NÚMERo DE SUJETOS, SEXO Y NÚMERO DE REACTORES POSITIVOS POR GRADO ESCOLAR. Tijuana, MÉxico, 1996

\begin{tabular}{lccc} 
& $\begin{array}{c}\text { Primer } \\
\text { año }\end{array}$ & $\begin{array}{c}\text { Grado escolar } \\
\text { Sexto } \\
\text { año }\end{array}$ & $\begin{array}{c}\text { Tercero de } \\
\text { secundaria }\end{array}$ \\
Número de sujetos & 341 & 356 & 434 \\
\hline \% del total & 30.1 & 31.4 & 38.4 \\
\hline Media de edad (años) & 6.69 & 11.9 & 14.7 \\
\hline \% de varones & 52.7 & 52.2 & 49.3 \\
\hline $\begin{array}{l}\text { \% de reactores } \\
\text { positivos }(\geq 10 \mathrm{~mm})\end{array}$ & 45.7 & 56.7 & 64.5 \\
\hline
\end{tabular}

\section{Cuadro II}

NúMERO Y PORCENTAJE dE REACTORES DE ACUERDO CON EL ANTECEDENTE DE INMUNIZACIÓN CON BCG. Tijuana, México, 1996

\begin{tabular}{lrrrrr}
$\begin{array}{l}\text { Inmunización } \\
\text { con BCG }\end{array}$ & Positivo & $\%$ & $\begin{array}{c}\text { Reactor } \\
\text { Negativo }\end{array}$ & $\%$ & Total \\
Sí & 556 & 59.7 & 376 & 40.3 & 932 \\
\hline No & 82 & 45.6 & 98 & 54.4 & 180 \\
\hline Total & 638 & 57.4 & 474 & 43 & I 112
\end{tabular}

\begin{tabular}{|c|c|c|c|c|}
\hline Resultados P & $\begin{array}{r}\text { CUA } \\
\text { LES DE }\end{array}$ & $\begin{array}{l}\text { O III } \\
\text { EGRESIÓN }\end{array}$ & & ÚLTIPLE \\
\hline Paso & $R^{2}$ & $R^{2}$ ajustada & B & $p$ \\
\hline Edad en años & 0.029 & 0.0283 & 0.17 & $<0.001$ \\
\hline Aplicación de BCG & 0.052 & 0.0500 & 0.08 & 0.003 \\
\hline
\end{tabular}

Variable dependiente: diámetro de induración al PPD en mm

reactores positivos $(45.6 \%)$ sigue siendo extremadamente alta. Los resultados de nuestra encuesta sólo son extrapolables a los escolares de la ciudad; los niños que no asisten a la escuela pueden tener un riesgo de infección tuberculosa diferente que los sujetos que sí lo hacen. Sin embargo, el único método práctico de seleccionar una muestra de niños para una encuesta tuberculínica que sea representativa del área en estudio es por medio de la selección de grupos de niños más que de individuos. El utilizar grupos de escuelas como unidades de muestreo se considera como válido si por 
lo menos $60 \%$ de los niños asiste a la escuela en esa región. ${ }^{10}$ En una encuesta realizada con anterioridad en Tijuana se estimó que más de $90 \%$ de los niños asisten o han asistido a la escuela. ${ }^{11}$

Algunos autores ${ }^{3,12}$ proponen calcular el riesgo de infección anual de tuberculosis (porcentaje de sujetos en una población que se infectan durante el periodo de un año) a partir de los resultados de una encuesta única; nosotros consideramos que este cálculo, para ofrecer resultados fidedignos, debe basarse necesariamente en la comparación de dos o más encuestas tuberculínicas en una misma cohorte.

Un factor que puede explicar, por lo menos parcialmente, la mayor prevalencia de reactores positivos observados en nuestro estudio en comparación con informes previos en la literatura nacional, es el hecho de que utilizamos PPD 5TU, mientras que en todas las demás encuestas tuberculínicas notificadas en nuestro país se ha utilizado tuberculina RT-23 de 2TU. Un informe reciente de Molina-Gamboa y colaboradores muestra que la sensibilidad del PPD RT-23 es tan sólo de un 57\% de la alcanzada con PPD 5TU, y ellos consideran que el uso de tuberculina RT-23 de 2TU ha llevado a una subestimación sistemática de la prevalencia de infección en México; de hecho recomiendan que tanto la prevalencia como el riesgo anual de infección tuberculosa en nuestro país sean revaluados a través de nuevas encuestas utilizando tuberculina 5TU. ${ }^{13}$

A pesar de que los sujetos nacidos fuera de Tijuana han residido en esta ciudad un periodo de tiempo significativamente menor que los sujetos nativos, el porcentaje de reactores positivos en ambos subgrupos es virtualmente idéntico; esto sugiere que los factores que favorecen la adquisición de la infección son similares en otras regiones de la República mexicana, o como hipótesis alternativa, que el riesgo de infección en Tijuana es tan elevado, que los sujetos de nuevo arribo, como grupo, alcanzan la misma prevalencia de infección que los nativos en un lapso más breve.

No tenemos una explicación satisfactoria a la proporción de reactores positivos significativamente mayor en el grupo de sujetos del sexo femenino, ya que el grado de cobertura con BCG es similar para ambos sexos, y no encontramos evidencia que sugiera un grado diferente de exposición u otros factores de riesgo específicos para adquisición de infección tuberculosa para este subgrupo.

La interpretación de los resultados de una prueba de tuberculina en sujetos inmunizados con BCG es motivo de controversia. La reactividad a la tuberculina después de la aplicación de BCG se ha informado con valores que van desde 0 hasta $19 \mathrm{~mm}$ de induración, ${ }^{14}$ ésta depende de varios factores que incluyen la dosis infectante de BCG, la vía de administración, la cepa y, especialmente, la edad del sujeto al momento de la inmunización. ${ }^{15}$ La aplicación de BCG en México es obligatoria en el recién nacido. ${ }^{5}$ Se ha descrito ampliamente que la reactividad a la tuberculina causada por la BCG, especialmente en los sujetos inmunizados al nacer, disminuye con el paso de los años y difícilmente persistirá por más de 10 años después de la inmunización en ausencia de infección natural por M. tuberculosis. . $^{14,16,18}$ Por esta razón, se ha recomendado que se considere a todos los sujetos con una reacción a la tuberculina $>10$ $\mathrm{mm}$ como infectado con M. tuberculosis, aun cuando dicho sujeto haya sido inmunizado con BCG, especialmente si fue inmunizado al nacer. ${ }^{14}$

La proporción de reactores positivos en nuestra muestra fue significativamente mayor en el grupo de sujetos inmunizados con BCG que en el de los no inmunizados. Sin embargo, varios hallazgos en nuestro estudio sugieren que esta diferencia en la reactividad no debe ser atribuida totalmente al efecto de la BCG porque: a) la proporción de reactores positivos entre las mujeres es significativamente mayor que entre los varones, a pesar de que el grado de cobertura con BCG fue el mismo para ambos grupos; b) el diámetro promedio de induración de los reactores positivos fue virtualmente idéntico cuando los sujetos se clasificaron por antecedente de inmunización (esto es, la magnitud de la reacción fue la misma, sin importar si habían sido inmunizados o no); c) la proporción de reactores positivos en el primer grado fue idéntica en sujetos inmunizados y no inmunizados (46.4 vs $47.7 \%$, respectivamente), pero para el tercer año de secundaria, el grupo de sujetos inmunizados presentaba $20 \%$ más reactores positivos que el grupo de no inmunizados $(69.7$ vs $49.9 \%$ ); y por último, d) el diámetro de induración a la tuberculina en nuestra muestra está positivamente correlacionado con la edad del sujeto (esto es, a mayor edad del sujeto mayor diámetro de induración), a pesar del incremento sincrónico del intervalo entre la edad al momento de la inmunización y la edad al momento de aplicar la prueba tuberculínica (intervalo promedio 9.31 años). Estos dos últimos hallazgos sugieren que la reactividad a la tuberculina en nuestra muestra está más relacionada con una infección natural por $M$. tuberculosis que con el efecto de la inmunización con BCG; la inmunización con BCG no evita la infección natural con micobacterias, en el mejor de los casos previene su diseminación, ${ }^{14}$ quizá la inmunización con BCG favorezca una respuesta de hiperergia más intensa a la tuberculina cuando el sujeto se infecta con $M$. tuberculosis (la infección natural ejercería un efecto de refuerzo o booster en un sujeto previamente inmunizado con BCG); 
esto explicaría el hallazgo paradójico de una mayor respuesta a la tuberculina a mayor edad, a pesar de un intervalo de casi 10 años en promedio entre la inmunización y la aplicación de la prueba tuberculínica.

Podemos concluir con base en nuestros hallazgos que la prevalencia de infección tuberculosa en la ciudad de Tijuana es extremadamente alta y muy por encima de los valores esperados de acuerdo con los informes de encuestas tuberculínicas previas realizadas en otras regiones de la República mexicana.

\section{Referencias}

I. Bloom BR, Murray CJL. Tuberculosis: Commentary on a reemergent killer. Science 1992;257:1055-1064.

2. Olvera-Castillo $R$, Jiménez-Galán A, Rodríguez-Filigrana S. Sensibilidad tuberculínica en población rural de Jalisco. Rev INER 1993;6:8I-87.

3. Gloyd S, López JL, Mercado FJ, Durning J. Riesgo de infección por Mycobacterium tuberculosis en Jalisco, México. Bol Oficina Sanit Panam | $991 ;$ | | I:393-40|.

4. Cauhthen GM, Pio A Ten Dam HG. Annual risk of tuberculosis infection. Ginebra:World Health Organization/TB, 1988;88:154.

5. Villarreal H, Martínez L, Salazar MA, Pérez G, Urrueta J, Olvera R. El control de la tuberculosis en la Republica Mexicana. Neumol Cir Torax 1995; LIV:I3-20.

6. XV Ayuntamiento de Tijuana. Tijuana: compromiso de todos. Plan Municipal de Desarrollo 1996-1998.Tijuana, Gobierno Municipal de Tijuana, 1996.

7. Laniado-Laborín R, Cárdenas-Moreno RP,Alvarez-Cerro M.Tijuana zona endémica de infección por Coccidioides immitis. Salud Publica Mex 1991;33:235-239.
8. American Thoracic Society. Diagnostic standards and classification of tuberculosis. Am Rev Respir Dis 1990;142:725-735.

9. Bouros D, Zeros G, Panaretos CH,Vassilatos CH, Siafakas N. Palpation vs. pen method for the measurement of skin tuberculin reaction (Mantoux test). Chest 1991;99:416-419.

I0. Styblo K. Methods used in the epidemiology of tuberculosis. Selected Papers 1991;24:10-13.

I I. Laniado-Laborín R, Moreno C,Vargas R, Deosaransingh K, Woodruff SI, Sallis JF. Los niños que trabajan en las calles de Tijuana. Perfil epidemiológico y prevalencia de experimentación con tabaco. Salud Publica Mex 1995;37: 149154.

12. Arnadottir T, Rieder HL, Trébucq A, Waaler HT. Guidelines for conducting tuberculin skin test surveys in high prevalence countries. Tuber Lung Dis 1996;77 suppl:I-20.

I3. Molina-Gamboa JD, Ponce de León-Rosales S, Rivera-Morales I, Romero C, Baez R, Huerta M et al. Evaluation of the sensitivity of RT-23 purified protein derivative for determining tuberculin reactivity in a group of health care workers. Clin Infect Dis 1994;19:784-786.

14. Center for Disease Control and Prevention. Recommendations and Reports. The role of BCG vaccine in the prevention and control of tuberculosis in the United States. MMWR Morb Mortal Wkly Rep 1996;45 suppl RR-4:I-I8.

15. Menzies R,Vissandjee B. Effect of bacille Calmette-Guérin vaccination on tuberculin reactivity. Am Rev Respir Dis 1992; 145:62I-625.

16. Pabst HF, Godel JC, Spady DW, McKechnie J, Grace M. Prospective trial of timing of bacillus Calmette-Guérin vaccination in canadian cree infants. Am Rev Respir Dis 1989; 140:1007-1011.

17. Joncas JH, Robitaille R, Gauthier T. Interpretation of the PPD skin test in BCG-vaccinated children. Can Med Assoc J 1975; I | 3: 127- 28.

18. Heyworth B. Delayed hypersensitivity to PPD-S following BCG vaccination in african children- an 18-month field study. Trans R Soc Trop Med Hyg 1977;7I:25I-253. 